\title{
IMPROVED PERFORMANCE OF SCS BASED SPECTRUM SENSING IN COGNITIVE RADIO USING DIFFERENT WINDOWS TECHNIQUES
}

\author{
Manish Pradhan ${ }^{1}$, Gaurav Gupta ${ }^{2}$ \\ ${ }^{1} M E$ Scholar, Department of ECE, MIT Ujjain, MP, India \\ ${ }^{2}$ Professor \& Head, Department of ECE, MIT Ujjain, MP, India
}

\begin{abstract}
Tremendous growth in current wireless networks raises the demand of more frequency spectrum, over the finite availability of spectrum resource. Although, the research has specifies that the available primary users (i.e. licensed user) has not occupying the channel all the time. The most effective technology known as Cognitive radio giving promises for a solution of under utilization of available frequency spectrum in wireless communication. In cognitive radio network two types of wireless user can be define as primary user and secondary user. Primary users have highest priority to utilize the available band of frequency and secondary user can utilize these services only when the channel is vacant by primary user and there will be no any interference. The optimization of this may be implemented by a smart technique such as cognitive radio, which is fully automated intelligent wireless sensor tool having capability to sense, learn \& adjust relevant operating parameters dynamically in radio atmosphere. This can be happen if we prefer the appropriate window technique to evaluate system parameter for sensing the availability of vacant band. We show that by comparing the different windows techniques, cognitive radios not only provide better spectrum opportunity but also provide the chance to huge number of wireless users.
\end{abstract}

Keywords: Primary user, Secondary user, Spectrum Sensing and Window technique etc.

\section{INTRODUCTION}

The field of Wireless communication obtaining the rapid growing era for communications industry in just few decade back. As a successive outcome, the system of wireless has become more advantageous through multiple areas like wireless internet in cellular telephony, laptops, and tablets. In with respect to time the variety of application demands more and more number of vacant bands [1].

Cognitive radio configures a remarkable solution to reduce the challenge of underutilization through permitting a possible access of available radio spectrum. For the better outcome the spectrum sensing technique must attained the desired performance design by the Federal Communications Commission (FCC) to provide flexibility to primary users' communications through the possible interference occurred due to secondary users. Here with our experimental modal we have obtained merely better efficiency of spectrum sensing technique by using different windows technique for the detection of vacant band.

The initial workout about the cognitive radio was carried out by Mitola in 2000 which specify that CRs can perform the operation of sensing for its environment, adopt the available radio resources, the requirements of various users [2].

a) How to detect the spectrum and the status of primary users.

b) How to monitor and configure the available band to meet users QoS parameters. Such management functions include spectrum analysis and spectrum decision by priority algorithm.

c) How to allot the available spectrum resources fairly. d) How to handled communication during transition (handoff) of selected frequency channels.

\subsection{Spectrum Awareness}

One of major task in cognitive radio is to retrieve information about the spectrum utilization and availability of PUs as well as SUs in an atmospheric area. The tradition approach to divide the net spectrum usage can be classified into three types as follows: (a) black spaces, (b) white spaces. Usually black spaces are consumed by local interferers having high power then the precious white spaces are available and free of any interferer by considering some environmental noise [3].

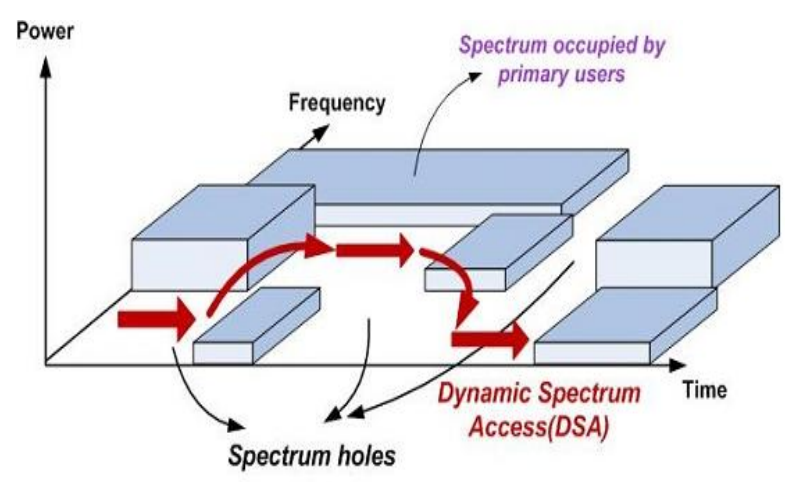

Fig 1: Spectrum availability showing with white space

The available white as well as gray spaces are termed as possible spectrum opportunities and represented as spectrum holes, which will be used for SUs. 


\subsection{Enabling Techniques}

For the proper implementation of cognitive radio systems, few of the enabling technologies are discussing here which include: Smart sensors, and software defined radio (SDR).

Smart Sensors also plays an important role to sense the surrounded radio environment. Few of them are showing as sensors of radio frequency receiver, sensitive microphone, biometric scanners (like fingerprint, iris, retina), digital camera, global positioning system (GPS). All such devices are used for spectrum sensing in cognitive radio. GPS will provide the information about location awareness.

The utilization of spectrum in every country is governed by the regulatory authority with regulation policies and it may vary on various country, time slot, operating software, and related hardware. The terminology of policy engine will assist the merging of different regulations by having a common platform of policies in specific downloadable software. While machine learning strategy will focuses on automatically sense and taking the right decisions through the available data. The advancement of each technology does play an important role in making the CRs more productive as well as accurate result oriented.

Now let we understand the backbone of enabling technology i.e. SDR abbreviated as software defined radio are the kind of software approach in which typical hardware implementation like signal generations their mixings, signals amplification, transmission, signal sensing etc. are possible using digital signal processor.

\section{PERFORMANCE ANALYSIS}

The core part of this proposed method has been with spectrum sensing then analysis through different windows techniques. So for spectrum sensing we are choosing spectral covariance method and then we approach for performance optimization.

\subsection{Spectrum Sensing through Spectral Covariance}

\section{Method:}

In this section our primary task is to perform the spectrum sensing of available signal in the specific frequency band.

\subsubsection{Requirements for Spectrum Sensing}

Here for the implementation we considering 20 different users, each have different frequency. For sampling we are considering sampling frequency $\mathrm{Fs}$ in $\mathrm{MHz}$ range and the bandwidth requirement for our simulation is about $1 \mathrm{MHz}$. We are also taking the signal to noise ratio in range of 30:1:30.

The problem of detection can be analyze by the given hypotheses as in (6):

$$
\text { H0: } z(n)=w(n),
$$

$$
\mathrm{H} 1: \mathrm{z}(\mathrm{n})=\mathrm{s}(\mathrm{n})+\mathrm{w}(\mathrm{n})
$$

Here $z(n)$ represents the baseband signal and $s(n)$ represents the signal component at receiver end similarly $\mathrm{w}(\mathrm{n})$ is shown the noise available in the received signal6. The signal part i.e. $s(n)$ can have a DC component $(\operatorname{sd}(n)=s d) \&$ an AC Component $(\mathrm{sa}(\mathrm{n}))$. Its estimation cab be taking out by considering the mean $\mathrm{E}[\mathrm{s}(\mathrm{n})]=\mathrm{sd}$ and the variance $\sigma 2 \mathrm{~s}$ $=\mathrm{E}[\mathrm{sa}(\mathrm{n}) 2]$. The noise samples $\mathrm{w}(\mathrm{n})$ is showing with zeromean value [6].

\subsubsection{Various Steps involved in Simulation}

For simplicity the description of each stage is mentioning here with relevant expression:

1) First we consider 20 numbers of different user are transmitting their signals in the range of $\mathrm{MHz}$ frequency. Each user have different frequency band.

2) Let transmit these generated signal into noisy environment for signal detection.

3) In next step we received the radio signal and then we down convert the $s(t)$ into a baseband complex signal $y(t)=$ $x(t) e^{-j 2 \pi f c t}$.

4) Next we use Low pass filter along with down sample $y(t)$ with sampling frequency $F s$ or $(1 / T s)$ for finding the sample function $z(n)$.

5) Here the spectrogram can be obtained by computing $z(n)$ 's with the squared magnitude of its short-time Fourier transform (STFT). The respective expression shown below:

$$
Z_{\tau}(k)=\frac{1}{N}\left|\sum_{n=0}^{\mathrm{N}-1} \mathrm{z}(\mathrm{n}+\tau \mathrm{N}) \mathrm{e}^{-\mathrm{j} 2 \pi \mathrm{nk} / \mathrm{N}}\right|^{2}
$$

where $N=256$ is the number of FFT points, and $\tau \in$ $\left\{0,1, \ldots ., N_{d}-1\right\}$ shows the index of the sensing window as we have taken rectangular window, $N_{\mathrm{d}}$ specifies the total number of sensing windows and $\mathrm{k} \in\{-N / 2, \ldots . ., 0, \ldots ., N / 2$ $-1\}$ is the frequency index. Hence, the FFT is calculated at every dwell time $(t s)$ for $N_{d}$ times.

6) Select components near DC according to

$$
M=\left[\begin{array}{ccc}
\mathrm{Z}_{\mathrm{o}}(-\mathrm{K}) & \cdots & \mathrm{Z}_{\mathrm{Nd}-1}(-\mathrm{K}) \\
\vdots & \cdots & \vdots \\
\mathrm{Z}_{\mathrm{o}}(\mathrm{K}) & \cdots & \mathrm{Z}_{\mathrm{Nd}-1}(\mathrm{~K})
\end{array}\right]
$$

In this matrix $K$ represents the index value of low pass filter cut off frequency $(B f)$ in FFT, i.e. $\mathrm{K}=[\mathrm{N} \cdot \mathrm{Bf} / \mathrm{F}]$. Now the matrix reduction requires a low pass filter, which can select the parameter of primary signal and reduces noise power.

7) Now calculate the sample covariance of $\mathrm{M}$ as $\mathrm{C}=\mathrm{cov}$ (M) and threshold T.

8) Compute the STFT Power and compare it with threshold value.

9) By comparing results check these hypotheses value and conclude the detection results.

10) simulate these results repeatedly at various SNR level i.e. from $-30: 1: 30$. 


\subsection{Optimization Approach}

In this section, our approach is directed towards the utilization of different windows technique for signal analysis in cognitive radio network so that the desired characteristics will meet to the expectation.

a) Windows Function: There are many windows function are available to synthesize the signal for various characteristics but it is not usual to allow all the available windows for spectrum analysis in cognitive radio. Out of all few of the methods are chosen which help us to provide more accurate results than any other else such available windows are [8]:

\section{Rectangular Window}

One of the simplest or Dirichlet window which is popularly known as rectangular window is similar to changing all but $\mathrm{N}$ set of values in available data sequence by putting zeros, for showing it like the available signal waveform instantly goes to on and off:

\section{Hamming Window}

The whole credit for developing such an innovative idea in to possible term is directed to Richard W. Hamming. Their invented window is used for signal optimization to reduce the maxima (side lobe), which is shows the height variation i.e. about one-fifth from the Hann window.

\section{Hann (Hanning) Window}

Another popular window derived by Julius von Hann is known as the Hanning (for being similar in name and form to the Hamming window), von Hann and the raised cosine window.

By putting these different windows one by one in to analysis and then by keen observing that, with each window there is variation in results. It is describe in the Simulation waveform waveforms.

\section{SIMULATION RESULTS}

The simulation of above mentioned approach has been implemented in Matlab. Here we used total three different windows for optimization as above mentioned. After getting multiple execution of the same program it is observed that there is difference is performance in each of the windows.

Here the figure 2 is representing the first execution of our proposed model in which we are looking that the available legend is showing the outcome with Hann, Hamming and Rectangular windows with color of green, red and blue respectively.

The Rectangular window is showing results very constantly and smoothly with minimum value of 0.4 at -30 SNR.

At a same SNR the Hann and Hamming window are showing the result of 0.4 and 0.25 respectively.

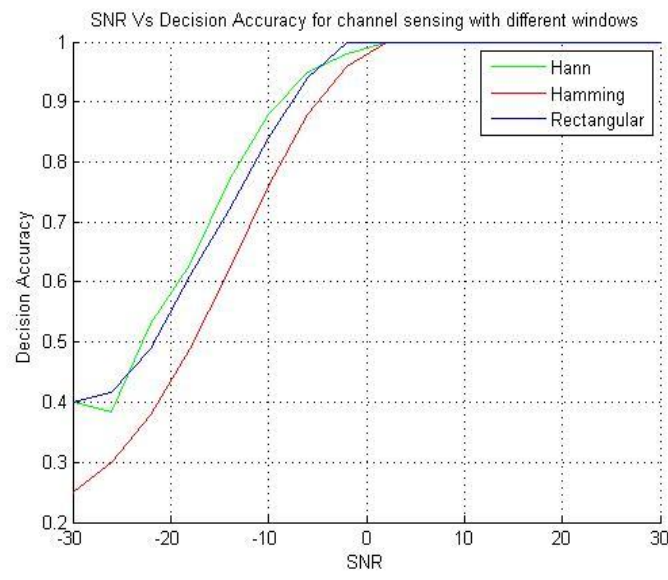

Fig 2: Simulation waveform result 1

In next execution as showing in figure 3 at same SNR we obtaining that hann window results vary from 0.25 to 0.45 while hamming window result decreases from 0.4 to 0.31 but the rectangular window does not affect the result much as it gradually increases.

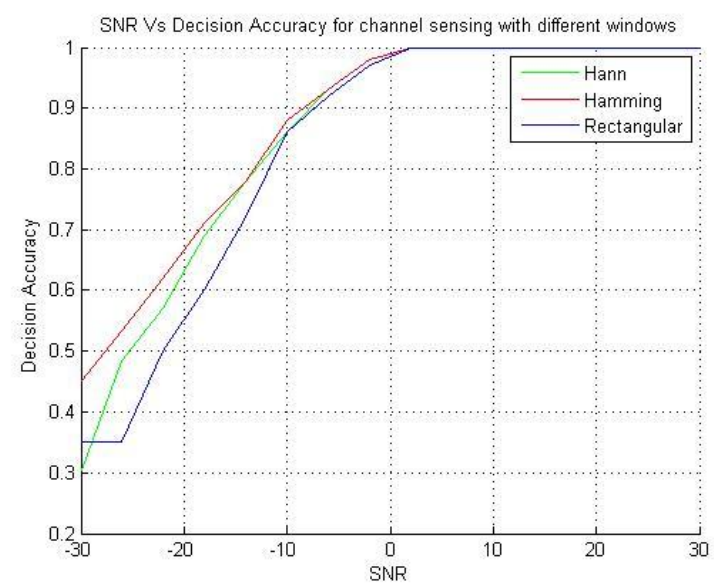

Fig 3: Simulation waveform result 2

Again in further simulation result we are getting that the rectangular window is showing the smooth variation with increasing of SNR while the hann and hamming does showing results with variation in every execution as these difference can easily see in figure 4.

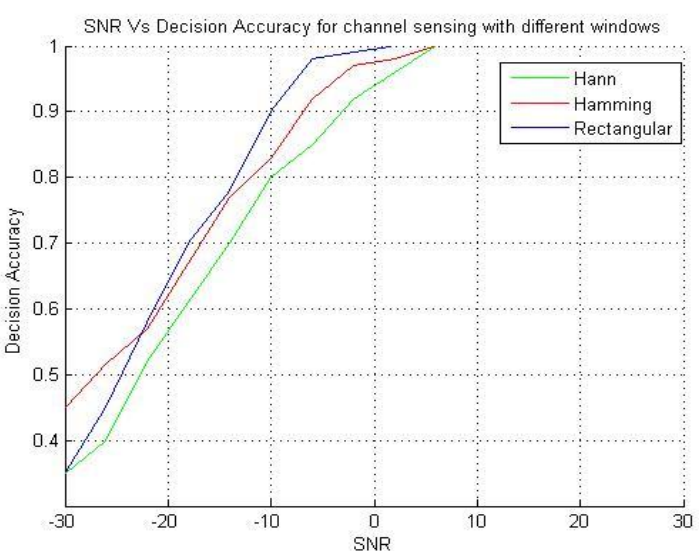

Fig 4: Simulation waveform result 3 
So by comparing all execution results it is well summarized that the rectangular window giving best possible results in multiple execution. And also it is increasing the decision accuracy gradually.

\section{CONCLUSION}

In this paper we proposed a model with the utilization of different windows to obtained high performance in cognitive radio. Because this technology enabling the solution to fill the gap between demand and supply in radio frequency for wireless user. With respect to time it is very immerging technology which is providing one of the best utilization of available radio spectrum. Many scientist and researchers are continuously working towards the advancement in the field of wireless communication. Our proposed model is surely enhancing the performance of radio detection in spectrum sensing technique to boost the utilization of cognitive radio.

\section{REFERENCES}

[1]. FCC, "FCC spectrum policy task force report, ET Docket no. 02-155," Nov. 2002.

[2]. Rozeha A Rashid, Norsheila Fisal "ISSUES OF SPECTRUM SENSING IN COGNITIVE RADIO BASED SYSTEMS"

[3]. S. Haykin, "Cognitive radio: Brain-empowered wireless communications," IEEE Journal of Selected Areas in Communications, vol. 23, no. 2, pp. 201-220, Feb. 2005.

[4]. S. Haykin, David J. Thomson, Jefeerey H. Reed "Spectrum sensing for cognitive radio," Processing of IEEE, vol. 97, No.5, May 2009.

[5]. Jaeweon Kim, Jeffrey G. Andrewa "Sensitive White Space Detection With Spectral Covariance Sensing" IEEE Transaction on Wireless Communication 2010.

[6]. Manish Pradhan, Gaurav Gupta "Performance Evaluation Of Spectral Covariance Based Spectrum Sensing Technique In Cognitive Radio". INTERNATIONAL JOURNAL OF SCIENCE, ENGINEERING AND TECHNOLOGY- www.ijset.in ISSN: 2348-4098 VOLUME 2 ISSUE 7 SEP-OCT 2014

[7]. "Efficient Spectrum Sensing for Cognitive Radio Networks via Joint Optimization of Sensing Threshold and Duration" Ling Luo and Sumit Roy IEEE TRANSACTIONS ON COMMUNICATIONS, VOL. 60, NO. 10, OCTOBER 2012.

[8]. Shushank Dogra, Narinder Sharma "Comparison of Different Techniques to Design of Filter" International Journal of Computer Application (0975-8887) Volume 97No.1, July 2014. 